\title{
OTULIN deficiency causes auto-inflammatory syndrome
}

\author{
Cell Research (2016) 26:1176-1177. doi:10.1038/cr.2016.113; published online 30 September 2016
}

Ubiquitin chains assembled via the N-terminal methionine (Met1 or linear ubiquitin), conjugated by the linear ubiquitin chain assembly complex (LUBAC), participate in NF- $\kappa B-d e p e n d e n t$ inflammatory signaling and immune responses. $A$ recent report in Cell finds that OTULIN, a deubiquitinase that selectively cleaves Met1-linked ubiquitin chains, is essential for restraining inflammation in vivo.

Ubiquitin signaling controls activation of nuclear factor- $\kappa \mathrm{B}(\mathrm{NF}-\mathrm{\kappa} B)$ and inflammatory responses upon engagement of pattern recognition receptors and cytokine receptors such as tumor necrosis factor (TNF) receptor 1 (TNFR1). Ubiquitin chains linked via lysine 63 (Lys63-Ub) and methionine 1 (Met1-Ub) are formed on protein substrates after receptor activation and coordinate activation of downstream kinase complexes, leading to NF- $\mathrm{kB}$ dependent transcription [1]. Met1-Ub chains are assembled by the linear ubiquitin chain assembly complex (LUBAC), composed of HOIP, HOIL-1, and SHARPIN [1]. LUBAC function is regulated by the deubiquitinases (DUBs) OTULIN [2-4] and CYLD [5-7], which both associate with the catalytic subunit HOIP [5-8].

Previous studies have revealed that OTULIN exclusively hydrolyzes Met1$\mathrm{Ub}$, prevents accumulation of Met1-Ub on LUBAC components under basal conditions, and restricts ubiquitination of LUBAC substrates after receptor stimulation [2-4]. However, the contribution of OTULIN to regulation of physiological immune responses had not been investigated.
Damgaard et al. [9] now reveal that a loss-of-function mutation in OTULIN leads to an early-onset autoinflammatory disease termed ORAS (OTULINrelated autoinflammatory syndrome). The study identifies a homozygous missense mutation in OTULIN $(c .815 T>C$; p.Leu272Pro) in three affected individuals from a consanguineous family. The mutation renders OTULIN less stable and severely diminishes its DUB activity. Accordingly, isolated white blood cells from an ORAS patient exhibited elevated Met1-Ub levels as compared to healthy donor cells. ORAS patients presented with early-onset and severe systemic inflammation that can be managed with anti-TNF treatment [9].

To dissect the mechanisms leading to ORAS, Damgaard et al. developed several mouse models. Mixed bone marrow chimeras were generated in which OTULIN was ablated in half of the bone marrow cells. This led to severe weight loss in the mice accompanied by increased neutrophil infiltration and elevated serum levels of cytokines and chemokines. Strikingly, treating mice with anti-TNF, like in the ORAS patients, rescued most disease symptoms [9].

Interestingly, analysis of mice where OTULIN was ablated in T-cells, B-cells or in myeloid cells showed that ablation of OTULIN in B- and T-cells caused no overt phenotype whereas mice that lacked OTULIN in myeloid cells displayed enlarged lymphoid organs and liver, had immune cell infiltration in the liver, and increased cytokine levels in serum [9]. While this suggests that lymphoid cells rely less on OTULIN for $\mathrm{NF}-\mathrm{\kappa B}$ pathway regulation than myeloid cells, this is unexpected since previous studies have found that mutations affecting LUBAC activity profoundly impact B- and T-cell function [1]. Instead, Damgaard et al. found that HOIP and SHARPIN levels were strongly reduced, presumably due to protein destabilization, in OTULIN-deficient Band T-cells but not in the myeloid cells. This might explain why the absence of OTULIN in B- and T-cells did not result in an overt phenotype in mice.

Analysis of signaling in OTULINdeficient bone marrow-derived macrophages (BMDMs) showed, like in the ORAS patient samples, spontaneous accumulation of Met1-Ub. Additionally, OTULIN-deficient BMDMs displayed features of elevated baseline activity of NF- $\kappa \mathrm{B}$ signaling, which was not reduced by TNF-blocking antibodies. This implies that loss of OTULIN in BMDMs causes enhanced cell-autonomous NF$\kappa \mathrm{B}$ signaling, possibly triggered by the accumulation of Met1-Ub [9].

A separate recent study also identified patients with mutations in OTULIN [10]. In addition to the L272P mutation, the study identifies two other mutations in the OTU domain. Two out of three patients respond to anti-TNF treatment with the third patient being treated with anti-IL-1 $\beta$ (anakinra) and steroids. The analysis of patient samples show, in agreement with Damgaard et al., increased levels of Met1-Ub and increased cytokine production relative to cells from healthy donors [10].

A number of intriguing questions arise from the findings by Damgaard et al. [9]. For example, how does accumulation of Met1-Ub in OTULIN-ablated BMDMs activate NF- $\kappa B$ independently 


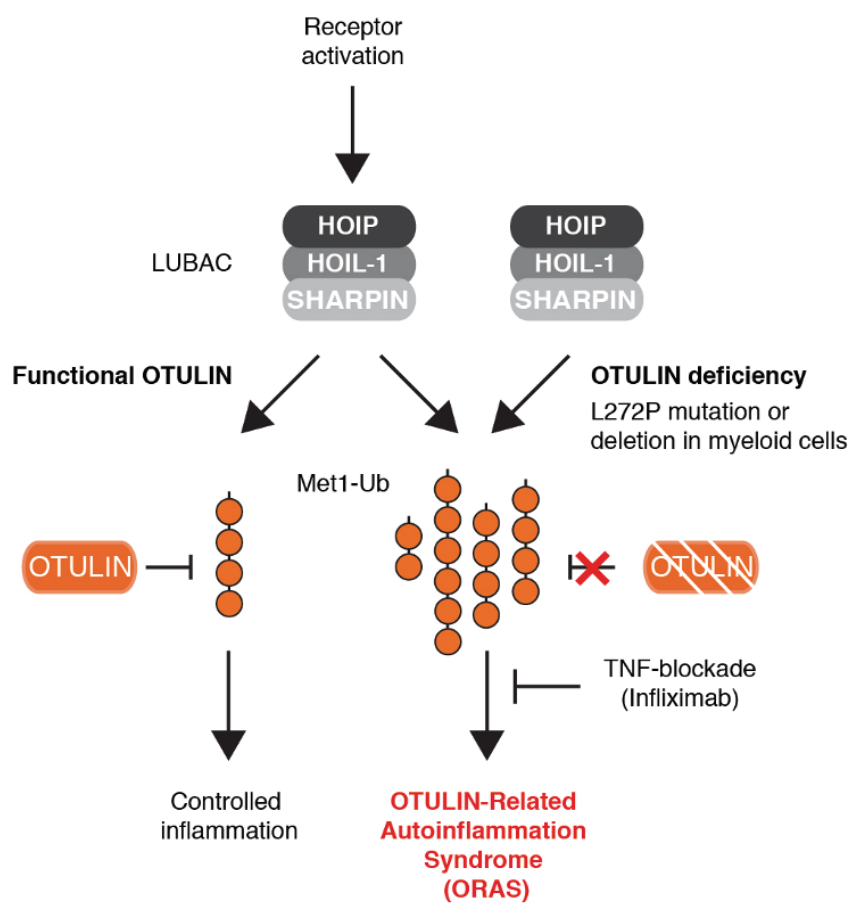

Figure 1 Activation of immune receptors triggers assembly of Met1-Ub by LUBAC that facilitates inflammation and under normal conditions is counter-balanced by the DUB OTULIN. Individuals with a loss-of-function mutation in OTULIN (L272P) or mice where OTULIN is deleted in myeloid cells accumulate Met1-Ub, possibly independently of receptor activation, and develop a severe autoinflammatory condition termed ORAS. Significantly, ORAS can be alleviated by the inhibition of TNF.

of receptor activation? Is the Met1-Ub further modified with other linkages, for example Lys63-Ub, to create a signaling platform similar to a receptor complex? It is tempting to speculate that OTULIN function under certain physiological conditions could be repressed to enable LUBAC-triggered NF- $\mathrm{\kappa B}$ signaling.

The observation that Met1-Ub accumulated in OTULIN-deficient cells rather than being disassembled by other cellular DUBs such as CYLD is intriguing because CYLD, like OTULIN, cleaves Met1-Ub and associates with HOIP [5-
7]. However, OTULIN and CYLD interact with distinct LUBAC complexes in a mutually exclusive manner [7], thus a possible explanation is that CYLD only regulates ubiquitin chains conjugated by a subset of LUBAC complexes. In addition to OTULIN and CYLD, the DUB A20 has also been implicated in Met1-Ub biology. A20 does not cleave Met1-Ub but instead binds the linkage via its Zinc finger 7, leading to stabilization of Met1-Ub in the TNFR1 complex $[1,7]$. In addition, the binding of A20 to Met1-Ub contributes to its ability to suppress NF- $\kappa \mathrm{B}$ activity, possibly by regulating NEMO/LUBAC signaling complexes $[11,12]$. Taken together, there is now overwhelming evidence that regulation of LUBAC function and Met1-Ub stability by DUBs is a central regulatory mechanism in NF- $\mathrm{kB}$ signaling and inflammation, which warrants further exploration of how OTULIN together with CYLD and A20 coordinate Met1-Ub signaling.

Berthe Katrine Fiil ${ }^{1}$, Mads Gyrd-Hansen ${ }^{1}$

${ }^{1}$ Ludwig Institute for Cancer Research, Nuffield Department of Medicine, University of Oxford, Old Road Campus Research Building, Oxford $O X 37 D Q, U K$

Correspondence: Mads Gyrd-Hansen

Tel: +44 (0)1865 617508

E-mail: mads.gyrd-hansen@ludwig.ox.ac.uk

\section{References}

1 Shimizu Y, Taraborrelli L, Walczak H. Immunol Rev 2015; 266:190-207.

2 Keusekotten K, Elliott PR, Glockner L, et al. Cell 2013; 153:1312-1326.

3 Fiil BK, Damgaard RB, Wagner SA, et al. Mol Cell 2013; 50:818-830.

4 Rivkin E, Almeida SM, Ceccarelli DF, et al. Nature 2013; 498:318-324.

5 Takiuchi T, Nakagawa T, Tamiya $\mathrm{H}$, et al. Genes Cells 2014; 19:254-272.

6 Hrdinka M, Fiil BK, Zucca M, et al. Cell Rep 2016; 14:2846-2858.

7 Draber P, Kupka S, Reichert M, et al. Cell Rep 2015; 13:2258-2272.

8 Elliott PR, Nielsen SV, Marco-Casanova P, et al. Mol Cell 2014; 54:335-348.

9 Damgaard RB, Walker JA, Marco-Casanova $\mathrm{P}$, et al. Cell 2016; 166:1215-1230.

10 Zhou Q, Yu X, Demirkaya E, et al. Proc Natl Acad Sci USA 2016; 113:10127-10132.

11 Verhelst K, Carpentier I, Kreike M, et al. EMBO J 2012; 31:3845-3855.

12 Tokunaga F, Nishimasu H, Ishitani R, et al. EMBO J 2012; 31:3856-3870. 